\title{
Note on a $q$-analogue of Stancu-Kantorovich operators
}

\author{
Ana Maria Acu, Dan Bărbosu, and Daniel Florin \\ Sofonea
}




\title{
NOTE ON A $q$-ANALOGUE OF STANCU-KANTOROVICH OPERATORS
}

\author{
ANA MARIA ACU, DAN BĂRBOSU, AND DANIEL FLORIN SOFONEA
}

Received 29 April, 2014

\begin{abstract}
The goal of this paper is to introduce new q-Stancu-Kantorovich operators and to study some of their approximation properties. A convergence theorem using the well known BohmanKorovkin criterion is proven and the rate of convergence involving the modulus of continuity is established. Furthermore, a Voronovskaja type theorem is also proven.
\end{abstract}

2010 Mathematics Subject Classification: 41A10; 41A25; 41A36

Keywords: q-Stancu-Kantorovich operators, modulus of continuity, rate of convergence, Voronovskaja theorem

\section{INTRODUCTION}

In 1987, A Lupaş [7] introduced and studied $q$ analogue of Bernstein operators, while in 1996 another generalization of these operators based on q-integers was introduced by Philips [13]. Generalizations of the Bernstein operators based on the q-integers attracted a lot of interest and were studied by a number of authors. During the last decade, the applications of q-calculus in the study of linear and positive operators were considered in $[5,6,8,10,12,14-16]$. The goal of the present paper is to introduce a Kantorovich variant of q-Stancu operators and investigate their approximation properties. Many generalizations and applications of the Stancu operators were considered in the last years $([1,2,11,15])$.

Before proceeding further, we mention some basic definitions and notations from $q$-calculus. Let $q>0$. For each nonnegative integer $k$, the $q$-integer $[k]$ and $q$ factorial $[k]$ ! are respectively defined by

$$
[k]:=\left\{\begin{array}{ll}
\frac{1-q^{k}}{1-q}, & q \neq 1, \\
k, & q=1,
\end{array},[k] !:= \begin{cases}{[k][k-1] \cdots[1],} & k \geq 1, \\
1, & k=0 .\end{cases}\right.
$$

For the integers $n, k$ satisfying $n \geq k \geq 0$, the $q$-binomial coefficients are defined by

$$
\left[\begin{array}{l}
n \\
k
\end{array}\right]:=\frac{[n] !}{[k] ![n-k] !} .
$$


We denote $(a+b)_{q}^{k}=\prod_{j=0}^{k-1}\left(a+b q^{j}\right)$.

The $q$-Jackson integral on the interval $[0, \mathrm{~b}]$ is defined as

$$
\int_{0}^{b} f(t) d_{q} t=(1-q) b \sum_{j=0}^{\infty} f\left(q^{j} b\right) q^{j}, 0<q<1,
$$

provided that sums converge absolutely. Suppose $0<a<b$. The $q$-Jackson integral on the interval $[a, b]$ is defined as

$$
\int_{a}^{b} f(t) d_{q} t=\int_{0}^{b} f(t) d_{q} t-\int_{0}^{a} f(t) d_{q} t, 0<q<1 .
$$

The clasical Bernstein-Kantorovich operators $B_{n}^{*}, n=1,2, \ldots$ are defined by

$$
\begin{aligned}
B_{n}^{*}(f, x) & :=(n+1) \sum_{k=0}^{n} p_{n, k}(x) \int_{k / n+1}^{k+1 / n+1} f(t) d t \\
& =\sum_{k=0}^{n} p_{n, k}(x) \int_{0}^{1} f\left(\frac{k+t}{n+1}\right) d t, f:[0,1] \rightarrow \mathbb{R},
\end{aligned}
$$

where $p_{n, k}(x)=\left(\begin{array}{l}n \\ k\end{array}\right) x^{k}(1-x)^{n-k}$.

In [3] the author introduced the $q$-analogue of the Bernstein-Kantorovich operators

$$
B_{n, q}(f ; x)=[n+1] \sum_{k=0}^{n} q^{-k} p_{n, k}(q ; x) \int_{[k] /[n+1]}^{[k+1] /[n+1]} f(t) d_{q} t,
$$

where $f \in C[0,1]$ and $p_{n, k}(q ; x)=\left[\begin{array}{l}n \\ k\end{array}\right] x^{k}(1-x)_{q}^{n-k}$.

In [8], inspired by (1.1), the authors introduced a $q$-type generalization of BernsteinKantorovich operators as follows

$$
B_{n, q}^{*}(f, x):=\sum_{k=0}^{n} p_{n, k}(q ; x) \int_{0}^{1} f\left(\frac{[k]+q^{k} t}{[n+1]}\right) d_{q} t,
$$

where $f \in C[0,1], 0<q<1$.

These operators verify:

Lemma 1 ([8]). For all $n \in \mathbb{N}, x \in[0,1]$ and $0<q<1$ we have

$$
\begin{aligned}
& B_{n, q}^{*}(1, x)=1, B_{n, q}^{*}(t, x)=\frac{2 q}{[2]} \frac{[n]}{[n+1]} x+\frac{1}{[2]} \frac{1}{[n+1]}, \\
& B_{n, q}^{*}\left(t^{2}, x\right)=\frac{q^{2}(q+2)}{[3]} \frac{[n][n-1]}{[n+1]^{2}} x^{2}+\frac{4 q+7 q^{2}+q^{3}}{[2][3]} \frac{[n]}{[n+1]^{2}} x+\frac{1}{[3]} \frac{1}{[n+1]^{2}} .
\end{aligned}
$$


In [15] two kinds of Kantorovich-type q-Bernstein-Stancu operators were introduced. The first version is defined using $q$-Jackson integral as follows

$$
S_{n, q}^{(\alpha, \beta)}(f, x)=([n+1]+\beta) \sum_{k=0}^{n} q^{-k} p_{n, k}(q ; x) \int_{\frac{[k]+\alpha}{[n+1]+\beta}}^{\frac{[k+1]+\alpha}{[n+1]+\beta}} f(t) d_{q} t .
$$

To guarantee the positivity of $S_{n, q}^{(\alpha, \beta)}$ in [15] a Riemann-type q-integral is considered (see [9]), with the following definition:

$$
\int_{a}^{b} f(t) d_{q}^{R} t=(1-q)(b-a) \sum_{j=0}^{\infty} f\left(a+(b-a) q^{j}\right) q^{j} .
$$

M.Y. Ren and X.M. Zeng redefine $S_{n, q}^{(\alpha, \beta)}(f ; x)$ by putting the Riemann-type qintegral into the operators instead of the q-Jackson integral as

$$
\tilde{S}_{n, q}^{(\alpha, \beta)}(f, x)=([n+1]+\beta) \sum_{k=0}^{n} q^{-k} p_{n, k}(q ; x) \int_{\frac{[k]+\alpha}{[n+1]+\beta}}^{\frac{[k+1]+\alpha}{[n+1]+\beta}} f(t) d_{q}^{R} t .
$$

The idea of using Riemann-type $q$-integral in the study of linear and positive operators, in order to guarantee the positivity, was first used by Ö. Dalmanoğlu and O. Doğru [4] for $q$-Bernstein-Kantorovich operators.

Inspired by (1.2) we introduce a q-type generalization of Stancu-Kantorovich operators as follows

$$
S_{n, q}^{*(\alpha, \beta)}(f, x)=\sum_{k=0}^{n} p_{n, k}(q ; x) \int_{0}^{1} f\left(\frac{[k]+q^{k} t+\alpha}{[n+1]+\beta}\right) d_{q} t,
$$

where $0 \leq \alpha \leq \beta$ and $f \in C[0,1]$.

\section{UNIFORM APPROXIMATION BY $q$-STANCU-KANTOROVICH OPERATORS}

Using the Bohman-Korovkin criterion we shall prove a convergence theorem for the q-Stancu-Kantorovich operators. First, we need the following two lemmas.

Lemma 2. For all $n \in \mathbb{N}, x \in[0,1]$ and $0<q<1$, we have

$$
S_{n, q}^{*(\alpha, \beta)}\left(t^{m}, x\right)=\frac{[n+1]^{m}}{([n+1]+\beta)^{m}} \sum_{\nu=0}^{m} \frac{\alpha^{m-v}}{[n+1]^{m-v}}\left(\begin{array}{c}
m \\
v
\end{array}\right) B_{n, q}^{*}\left(t^{\nu}, x\right) .
$$

Proof. By direct computation we obtain

$$
\begin{aligned}
& S_{n, q}^{*(\alpha, \beta)}\left(t^{m}, x\right)=\frac{[n+1]^{m}}{([n+1]+\beta)^{m}} \sum_{k=0}^{n} p_{n, k}(q ; x) \int_{0}^{1}\left(\frac{[k]+q^{k} t+\alpha}{[n+1]}\right)^{m} d_{q} t \\
& =\frac{[n+1]^{m}}{([n+1]+\beta)^{m}} \sum_{k=0}^{n} p_{n, k}(q ; x) \int_{0}^{1} \sum_{v=0}^{m}\left(\begin{array}{c}
m \\
v
\end{array}\right)\left(\frac{[k]+q^{k} t}{[n+1]}\right)^{v} \frac{\alpha^{m-v}}{[n+1]^{m-v}} d_{q} t
\end{aligned}
$$




$$
\begin{aligned}
& =\frac{[n+1]^{m}}{([n+1]+\beta)^{m}} \sum_{\nu=0}^{m} \frac{\alpha^{m-v}}{[n+1]^{m-v}}\left(\begin{array}{c}
m \\
v
\end{array}\right) \sum_{k=0}^{n} p_{n, k}(q ; x) \int_{0}^{1}\left(\frac{[k]+q^{k} t}{[n+1]}\right)^{v} d_{q} t \\
& =\frac{[n+1]^{m}}{([n+1]+\beta)^{m}} \sum_{\nu=0}^{m} \frac{\alpha^{m-v}}{[n+1]^{m-v}}\left(\begin{array}{c}
m \\
v
\end{array}\right) B_{n, q}^{*}\left(t^{v}, x\right) .
\end{aligned}
$$

Lemma 3. For all $n \in \mathbb{N}, x \in[0,1]$ and $0<q<1$, we have

$$
\begin{aligned}
S_{n, q}^{*(\alpha, \beta)}(1, x)=1, & S_{n, q}^{*(\alpha, \beta)}(t, x)=\frac{2 q}{[2]} \frac{[n]}{[n+1]+\beta} x+\frac{\alpha}{[n+1]+\beta}+\frac{1}{[2]([n+1]+\beta)}, \\
S_{n, q}^{*(\alpha, \beta)}\left(t^{2}, x\right)= & \frac{1}{([n+1]+\beta)^{2}}\left\{\frac{q^{2}(q+2)}{[3]}[n][n-1] x^{2}\right. \\
& \left.+\frac{q[n]}{[2]}\left(4 \alpha+\frac{4+7 q+q^{2}}{[3]}\right) x+\frac{2 \alpha}{[2]}+\frac{1}{[3]}+\alpha^{2}\right\} .
\end{aligned}
$$

Proof. Taking into account (2.1) and Lemma 1, by direct computation we obtain explicit formulas for $S_{n, q}^{*(\alpha, \beta)}\left(t^{i}, x\right), i=0,1,2$, as follows

$$
\begin{aligned}
S_{n, q}^{*(\alpha, \beta)}(1, x)= & B_{n, q}^{*}(1, x)=1 \\
S_{n, q}^{*(\alpha, \beta)}(t, x)= & \frac{[n+1]}{[n+1]+\beta}\left\{\frac{\alpha}{[n+1]} B_{n, q}^{*}(1, x)+B_{n, q}^{*}(t, x)\right\} \\
= & \frac{2 q}{[2]} \frac{[n]}{[n+1]+\beta} x+\frac{\alpha}{[n+1]+\beta}+\frac{1}{[2]([n+1]+\beta)}, \\
S_{n, q}^{*(\alpha, \beta)}\left(t^{2}, x\right)= & \frac{[n+1]^{2}}{([n+1]+\beta)^{2}}\left\{\frac{\alpha^{2}}{[n+1]^{2}} B_{n, q}^{*}(1, x)+\frac{2 \alpha}{[n+1]} B_{n, q}^{*}(t, x)+B_{n, q}^{*}\left(t^{2}, x\right)\right\} \\
= & \frac{1}{([n+1]+\beta)^{2}}\left\{\frac{q^{2}(q+2)}{[3]}[n][n-1] x^{2}\right. \\
& \left.+\frac{q[n]}{[2]}\left(4 \alpha+\frac{4+7 q+q^{2}}{[3]}\right) x+\frac{2 \alpha}{[2]}+\frac{1}{[3]}+\alpha^{2}\right\} .
\end{aligned}
$$

Lemma 4. For all $n \in \mathbb{N}, x \in[0,1]$ and $0<q<1$, we have

$$
\begin{aligned}
& S_{n, q}^{*(\alpha, \beta)}\left((t-x)^{2}, x\right) \leq \frac{2[n+1]^{2}}{([n+1]+\beta)^{2}}\left\{\frac{4}{[n]}\left(x(1-x)+\frac{1}{[n]}\right)+\left(\frac{\alpha}{[n+1]}-\frac{\beta}{[n+1]} x\right)^{2}\right\}, \\
& S_{n, q}^{*(\alpha, \beta)}\left((t-x)^{4}, x\right) \leq \frac{8[n+1]^{2}}{([n+1]+\beta)^{2}}\left\{\frac{C}{[n]^{2}}\left(x(1-x)+\frac{1}{[n]^{2}}\right)+\left(\frac{\alpha}{[n+1]}-\frac{\beta}{[n+1]} x\right)^{4}\right\},
\end{aligned}
$$

where $C$ is a positive absolute constant. 
Proof. The proof is based on the estimations of the second and fourth order central moments of the $q$-Bernstein-Kantorovich operators (Lemma 2.4 in [8]):

$$
\begin{aligned}
& B_{n, q}^{*}\left((t-x)^{2}, x\right) \leq \frac{4}{[n]}\left(x(1-x)+\frac{1}{[n]}\right), \\
& B_{n, q}^{*}\left((t-x)^{4}, x\right) \leq \frac{C}{[n]^{2}}\left(x(1-x)+\frac{1}{[n]^{2}}\right) .
\end{aligned}
$$

We have

$$
\begin{aligned}
& S_{n, q}^{*(\alpha, \beta)}\left((t-x)^{2}, x\right)=\sum_{k=0}^{n} p_{n, k}(q ; x) \int_{0}^{1}\left(\frac{[k]+\alpha+q^{k} t}{[n+1]+\beta}-x\right)^{2} d_{q} t \\
& =\frac{[n+1]^{2}}{([n+1]+\beta)^{2}} \sum_{k=0}^{n} p_{n, k}(q ; x) \int_{0}^{1}\left(\frac{[k]+q^{k} t}{[n+1]}-x+\frac{\alpha}{[n+1]}-\frac{\beta}{[n+1]} x\right)^{2} d_{q} t \\
& \leq \frac{2[n+1]^{2}}{([n+1]+\beta)^{2}}\left\{\sum_{k=0}^{n} p_{n, k}(q ; x) \int_{0}^{1}\left(\frac{[k]+q^{k} t}{[n+1]}-x\right)^{2} d_{q} t\right. \\
& \left.+\sum_{k=0}^{n} p_{n, k}(q ; x) \int_{0}^{1}\left(\frac{\alpha}{[n+1]}-\frac{\beta}{[n+1]} x\right)^{2} d_{q} t\right\} \\
& =\frac{2[n+1]^{2}}{([n+1]+\beta)^{2}}\left\{\frac{4}{[n]}\left(x(1-x)+\frac{1}{[n]}\right)+\left(\frac{\alpha}{[n+1]}-\frac{\beta}{[n+1]} x\right)^{2}\right\} .
\end{aligned}
$$

A similar calculus leads to

$$
\begin{aligned}
& S_{n, q}^{*(\alpha, \beta)}\left((t-x)^{4}, x\right) \leq \frac{8[n+1]^{2}}{([n+1]+\beta)^{2}}\left\{\sum_{k=0}^{n} p_{n, k}(q ; x) \int_{0}^{1}\left(\frac{[k]+q^{k} t}{[n+1]}-x\right)^{4} d_{q} t\right. \\
& \left.+\sum_{k=0}^{n} p_{n, k}(q ; x) \int_{0}^{1}\left(\frac{\alpha}{[n+1]}-\frac{\beta}{[n+1]} x\right)^{4} d_{q} t\right\} \\
& \leq \frac{8[n+1]^{2}}{([n+1]+\beta)^{2}}\left\{\frac{C}{[n]^{2}}\left(x(1-x)+\frac{1}{[n]^{2}}\right)+\left(\frac{\alpha}{[n+1]}-\frac{\beta}{[n+1]} x\right)^{4}\right\} .
\end{aligned}
$$

Theorem 1. Let $\left(q_{n}\right)_{n}, 0<q_{n}<1$ be a sequence satisfying the following conditions

$$
\lim _{n \rightarrow \infty} q_{n}=1, \lim _{n \rightarrow \infty} q_{n}^{n}=a, a \in[0,1) .
$$

Then for any $f \in C[0,1]$, the sequence $\left\{S_{n, q_{n}}^{*(\alpha, \beta)}(f, x)\right\}$ converges to $f$ uniformly on $[0,1]$. 
Proof. Using the Bohman-Korovkin theorem, it is sufficient to verify

$$
\lim _{n \rightarrow \infty} S_{n, q_{n}}^{*(\alpha, \beta)}\left(e_{i}, x\right)=x^{i}, i=0,1,2 \text {, uniformly on }[0,1] \text {. }
$$

If $q_{n} \rightarrow 1$, then $\lim _{n \rightarrow \infty}[n]=\infty$ and $\lim _{n \rightarrow \infty} \frac{[n]}{[n+1]+\beta}=1, \lim _{n \rightarrow \infty} \frac{[n][n-1]}{([n+1]+\beta)^{2}}=1$, hence (2.3) follows from Lemma 3.

\section{ThE RATE OF CONVERGENCE OF $q$-STANCU-KANTOROVICH OPERATORS}

In this section we will estimate the rate of convergence in terms of modulus of continuity. Furthermore, we calculate the rate of convergence in terms of the first modulus of continuity of the derivative of the function.

Recall that the first modulus of continuity of $f \in C[0, b]$ for $\delta>0$ is given by

$$
\omega(f, \delta)=\sup _{|y-x| \leq \delta}|f(y)-f(x)|, x, y \in[0, b] .
$$

Theorem 2. Let $\left(q_{n}\right)_{n}$ be a sequence satisfying conditions (2.2). Then

$$
\left|S_{n, q_{n}}^{*(\alpha, \beta)}(f, x)-f(x)\right| \leq 2 \omega\left(f ; \sqrt{\delta_{n}(x)}\right)
$$

for all $f \in C[0,1]$, where

$$
\delta_{n}(x)=S_{n, q_{n}}^{*(\alpha, \beta)}\left((t-x)^{2}, x\right)
$$

Proof. Since $S_{n, q_{n}}^{*(\alpha, \beta)}\left(e_{0}, \cdot\right)=e_{0}$, we have

$$
\begin{aligned}
\left|S_{n, q_{n}}^{*(\alpha, \beta)}(f, x)-f(x)\right| & =\left|\sum_{k=0}^{n} p_{n, k}\left(q_{n} ; x\right) \int_{0}^{1}\left[f\left(\frac{[k]+q_{n}^{k} t+\alpha}{[n+1]+\beta}\right)-f(x)\right] d_{q_{n}} t\right| \\
& \leq \sum_{k=0}^{n} p_{n, k}\left(q_{n} ; x\right) \int_{0}^{1}\left|f\left(\frac{[k]+q_{n}^{k} t+\alpha}{[n+1]+\beta}\right)-f(x)\right| d_{q_{n}} t .
\end{aligned}
$$

Using the following well known property of modulus of continuity

$$
|f(t)-f(x)| \leq \omega(f ; \delta)\left(\frac{|t-x|}{\delta}+1\right), \delta>0
$$

we get

$$
\begin{aligned}
& \left|S_{n, q_{n}}^{*(\alpha, \beta)}(f, x)-f(x)\right| \leq \sum_{k=0}^{n} p_{n, k}\left(q_{n} ; x\right) \int_{0}^{1}\left(\frac{1}{\delta}\left|\frac{[k]+q_{n}^{k} t+\alpha}{[n+1]+\beta}-x\right|+1\right) \omega(f, \delta) d_{q_{n}} t \\
& =\omega(f ; \delta)+\frac{\omega(f ; \delta)}{\delta} \sum_{k=0}^{n} p_{n, k}\left(q_{n} ; x\right) \int_{0}^{1}\left|\frac{[k]+q_{n}^{k} t+\alpha}{[n+1]+\beta}-x\right| d_{q_{n}} t .
\end{aligned}
$$


By the Cauchy-Schwarz inequality it follows

$$
\begin{aligned}
& \sum_{k=0}^{n} p_{n, k}\left(q_{n} ; x\right) \int_{0}^{1}\left|\frac{[k]+q_{n}^{k} t+\alpha}{[n+1]+\beta}-x\right| d_{q_{n} t} \\
& \leq \sum_{k=0}^{n} p_{n, k}\left(q_{n} ; x\right)\left\{\int_{0}^{1}\left(\frac{[k]+q_{n}^{k} t+\alpha}{[n+1]+\beta}-x\right)^{2} d_{q_{n}} t\right\}^{1 / 2}\left\{\int_{0}^{1} d_{q_{n}} t\right\}^{1 / 2} \\
& =\sum_{k=0}^{n}\left\{p_{n, k}\left(q_{n} ; x\right)\right\}^{1 / 2}\left\{p_{n, k}\left(q_{n} ; x\right) \int_{0}^{1}\left(\frac{[k]+q_{n}^{k} t+\alpha}{[n+1]+\beta}-x\right)^{2} d_{q_{n}} t\right\}^{1 / 2} \\
& \leq\left\{S_{n, q_{n}}^{*(\alpha, \beta)}\left((t-x)^{2}, x\right)\right\}^{1 / 2} .
\end{aligned}
$$

Therefore

$$
\left|S_{n, q_{n}}^{*(\alpha, \beta)}(f, x)-f(x)\right| \leq\left(1+\frac{1}{\delta} \sqrt{S_{n, q_{n}}^{*(\alpha, \beta)}\left((t-x)^{2}, x\right)}\right) \omega(f, \delta) .
$$

But, by Lemma 4 and conditions (2.2), $\lim _{n \rightarrow \infty} S_{n, q_{n}}^{*(\alpha, \beta)}\left((t-x)^{2}, x\right)=0$. So, letting $\delta_{n}(x)=\sqrt{S_{n, q_{n}}^{*(\alpha, \beta)}\left((t-x)^{2}, x\right)}$ and taking $\delta=\sqrt{\delta_{n}(x)}$, we get the result.

Denote

$$
\operatorname{Lip}_{M}(\gamma)=\left\{f \in C[0,1],|f(t)-f(x)| \leq M|t-x|^{\gamma}\right\}, 0<\gamma \leq 1 .
$$

The next result give the rate of convergence of operators $S_{n, q_{n}}^{*(\alpha, \beta)}$ in terms of the Lipschitz class.

Theorem 3. Let $\left(q_{n}\right)_{n}$ be a sequence satisfying conditions (2.2) and $f \in \operatorname{Lip} M(\gamma)$. Then

$$
\left|S_{n, q_{n}}^{*(\alpha, \beta)}(f, x)-f(x)\right| \leq M\left(\delta_{n}(x)\right)^{\gamma / 2}
$$

where $\delta_{n}(x)=S_{n, q_{n}}^{*(\alpha, \beta)}\left((t-x)^{2}, x\right)$.

Proof. Since $S_{n, q_{n}}^{*(\alpha, \beta)}\left(e_{0}, \cdot\right)=e_{0}$ and $f \in \operatorname{Li} p_{M}(\gamma)$, we have

$$
\begin{aligned}
\left|S_{n, q_{n}}^{*(\alpha, \beta)}(f, x)-f(x)\right| & \leq \sum_{k=0}^{n} p_{n, k}\left(q_{n} ; x\right) \int_{0}^{1}\left|f\left(\frac{[k]+q_{n}^{k} t+\alpha}{[n+1]+\beta}\right)-f(x)\right| d_{q_{n}} t \\
& \leq M \sum_{k=0}^{n} p_{n, k}\left(q_{n} ; x\right) \int_{0}^{1}\left|\frac{[k]+q_{n}^{k} t+\alpha}{[n+1]+\beta}-x\right|^{\gamma} d_{q_{n}} t .
\end{aligned}
$$


Appling the Hölder's inequality with $p=\frac{2}{\gamma}$ and $q=\frac{2}{2-\gamma}$, we get

$$
\begin{aligned}
& \left|S_{n, q_{n}}^{*(\alpha, \beta)}(f, x)-f(x)\right| \\
& \leq M \sum_{k=0}^{n} p_{n, k}\left(q_{n} ; x\right)\left\{\int_{0}^{1}\left(\frac{[k]+q_{n}^{k} t+\alpha}{[n+1]+\beta}-x\right)^{2} d_{q_{n}} t\right\}\left\{\int_{0}^{1} d_{q_{n}} t\right\}^{\frac{2-\gamma}{2}} \\
& =M \sum_{k=0}^{n} p_{n, k}\left(q_{n} ; x\right)\left\{\int_{0}^{1}\left(\frac{[k]+q_{n}^{k} t+\alpha}{[n+1]+\beta}-x\right)^{2} d_{q_{n}} t\right\}^{\gamma / 2} \\
& =M \sum_{k=0}^{n}\left(p_{n, k}\left(q_{n} ; x\right)\right)^{\frac{2-\gamma}{2}}\left\{p_{n, k}\left(q_{n} ; x\right) \int_{0}^{1}\left(\frac{[k]+q_{n}^{k} t+\alpha}{[n+1]+\beta}-x\right)^{2} d_{q_{n}} t\right\}^{\gamma / 2} \\
& \leq M\left\{\sum_{k=0}^{n} p_{n, k}\left(q_{n} ; x\right) \int_{0}^{1}\left(\frac{[k]+q_{n}^{k} t+\alpha}{[n+1]+\beta}-x\right)^{2} d_{q_{n}} t\right\}^{\gamma / 2} \\
& =M\left\{S_{n, q_{n}}^{*(\alpha, \beta)}\left((t-x)^{2}, x\right)\right\}^{\gamma / 2} .
\end{aligned}
$$

Theorem 4. Let $\left(q_{n}\right), 0<q_{n}<1$ be a sequence satisfying the conditions (2.2). Then for any $f \in C^{1}[0,1]$ and $x \in[0,1]$ we have

$$
\left|S_{n, q_{n}}^{*(\alpha, \beta)}(f ; x)-f(x)\right| \leq \lambda_{n}(x)\left|f^{\prime}(x)\right|+2 \sqrt{\delta_{n}(x)} \omega\left(f^{\prime}, \sqrt{\delta_{n}(x)}\right),
$$

where $\lambda_{n}(x)=S_{n, q_{n}}^{*(\alpha, \beta)}(t-x, x)$ and $\delta_{n}(x)=S_{n, q_{n}}^{*(\alpha, \beta)}\left((t-x)^{2}, x\right)$.

Proof. Let $f \in C^{1}[0,1]$. For any $t \in[0,1], x \in[0,1]$, we have

$$
f(t)-f(x)=f^{\prime}(x)(t-x)+\int_{x}^{t}\left(f^{\prime}(u)-f^{\prime}(x)\right) d u,
$$

so we get

$$
\begin{aligned}
S_{n, q_{n}}^{*(\alpha, \beta)}(f(t)- & f(x), x) \\
& =f^{\prime}(x) S_{n, q_{n}}^{*(\alpha, \beta)}(t-x, x)+S_{n, q_{n}}^{*(\alpha, \beta)}\left(\int_{x}^{t}\left(f^{\prime}(u)-f^{\prime}(x)\right) d u, x\right) .
\end{aligned}
$$

Using the well known property of modulus of continuity (3.1) we have

$$
\left|\int_{x}^{t}\right| f^{\prime}(u)-f^{\prime}(x)|d u| \leq \omega\left(f^{\prime} ; \delta\right)\left[\frac{(t-x)^{2}}{\delta}+|t-x|\right] .
$$


Therefore

$$
\begin{aligned}
\left|S_{n, q_{n}}^{*(\alpha, \beta)}(f, x)-f(x)\right| \leq & \left|f^{\prime}(x)\right| \cdot\left|S_{n, q_{n}}^{*(\alpha, \beta)}(t-x, x)\right| \\
& +\omega\left(f^{\prime} ; \delta\right)\left\{\frac{1}{\delta} S_{n, q_{n}}^{*(\alpha, \beta)}\left((t-x)^{2}, x\right)+S_{n, q_{n}}^{*(\alpha, \beta)}(|t-x|, x)\right\} .
\end{aligned}
$$

Using Cauchy-Schwartz inequality, we obtain

$$
\begin{aligned}
S_{n, q_{n}}^{*(\alpha, \beta)}(|t-x|, x) & \leq \sqrt{S_{n, q_{n}}^{*(\alpha, \beta)}(1, x)} \cdot \sqrt{S_{n, q_{n}}^{*(\alpha, \beta)}\left((t-x)^{2}, x\right)}, \\
S_{n, q_{n}}^{*(\alpha, \beta)}\left((t-x)^{2}, x\right) & \leq \sqrt{S_{n, q_{n}}^{*(\alpha, \beta)}\left((t-x)^{2}, x\right)} \cdot \sqrt{S_{n, q_{n}}^{*(\alpha, \beta)}\left((t-x)^{2}, x\right)},
\end{aligned}
$$

so we have

$$
\begin{aligned}
\mid S_{n, q_{n}}^{*(\alpha, \beta)}(f, x) & -f(x)|\leq| f^{\prime}(x)|\cdot| S_{n, q_{n}}^{*(\alpha, \beta)}(t-x, x) \mid \\
& +\omega\left(f^{\prime}, \delta\right)\left\{\frac{1}{\delta} \sqrt{\left.S_{n, q_{n}}^{*(\alpha, \beta)}\left((t-x)^{2}, x\right)\right)}+1\right\} \sqrt{S_{n, q_{n}}^{*}\left((t-x)^{2}, x\right)} \\
& =\left|f^{\prime}(x)\right| \lambda_{n}(x)+\omega\left(f^{\prime}, \delta\right) \cdot\left\{\frac{1}{\delta} \sqrt{\delta_{n}(x)}+1\right\} \sqrt{\delta_{n}(x)} .
\end{aligned}
$$

Choosing $\delta=\sqrt{\delta_{n}(x)}$, we find relation (3.2).

\section{A VORONOVSKAYA THEORM FOR $q$-STANCU-KANTOROVICH OPERATORS}

In this section we shall establish a Voronovskaja type theorem for $S_{n, q}^{*(\alpha, \beta)}$. First, we need the auxiliary result contained in the following lemma.

Lemma 5. Assume that $0<q_{n}<1, q_{n} \rightarrow 1$ and $q_{n}^{n} \rightarrow a$, $a \in[0,1)$ as $n \rightarrow \infty$. Then we have

$$
\begin{aligned}
\lim _{n \rightarrow \infty}[n] S_{n, q_{n}}^{*(\alpha, \beta)}(t-x ; x) & =-\frac{1+a+2 \beta}{2} x+\alpha+\frac{1}{2}, \\
\lim _{n \rightarrow \infty}[n] S_{n, q_{n}}^{*(\alpha, \beta)}\left((t-x)^{2} ; x\right) & =-\frac{2 a+1}{3} x^{2}+x .
\end{aligned}
$$

Proof. To prove the lemma we use the formulas for $S_{n, q_{n}}^{*(\alpha, \beta)}\left(t^{i}, x\right), i=0,1,2$ given in Lemma 3.

$$
\begin{aligned}
& \lim _{n \rightarrow \infty}[n] S_{n, q_{n}}^{*(\alpha, \beta)}(t-x ; x) \\
& =\lim _{n \rightarrow \infty}[n]\left\{\left(\frac{2 q_{n}}{[2]} \frac{[n]}{[n+1]+\beta}-1\right) x+\frac{\alpha}{[n+1]+\beta}+\frac{1}{[2]([n+1]+\beta)}\right\} \\
& =\lim _{n \rightarrow \infty}\left\{\frac{[n]}{[2]([n+1]+\beta)}\left(-1-q_{n}^{n+1}-[2] \beta\right) x+\frac{\alpha[n]}{[n+1]+\beta}+\frac{[n]}{[2]([n+1]+\beta)}\right\} \\
& =-\frac{1+a+2 \beta}{2} x+\alpha+\frac{1}{2} .
\end{aligned}
$$




$$
\begin{aligned}
& \lim _{n \rightarrow \infty}[n] S_{n, q_{n}}^{*(\alpha, \beta)}\left((t-x)^{2} ; x\right)=\lim _{n \rightarrow \infty}[n]\left\{S_{n, q_{n}}^{*(\alpha, \beta)}\left(t^{2}, x\right)-x^{2}-2 x S_{n, q_{n}}^{*(\alpha, \beta)}(t-x, x)\right\} \\
&=\lim _{n \rightarrow \infty}[n]\left(\frac{q_{n}^{2}\left(q_{n}+2\right)[n][n-1]}{[3]([n+1]+\beta)^{2}}-1\right) x^{2} \\
&+\lim _{n \rightarrow \infty} \frac{[n]^{2}}{[2]([n+1]+\beta)^{2}}\left(4 q_{n} \alpha+\frac{4 q_{n}+7 q_{n}^{2}+q_{n}^{3}}{[3]}\right) x \\
&+\lim _{n \rightarrow \infty}[n]\left\{\frac{\alpha^{2}}{([n+1]+\beta)^{2}}+\frac{2 \alpha}{[2]([n+1]+\beta)^{2}}+\frac{1}{[3]([n+1]+\beta)^{2}}\right\} \\
&-\lim _{n \rightarrow \infty} 2 x[n] S_{n, q_{n}}^{*(\alpha, \beta)}(t-x, x) \\
&= \lim _{n \rightarrow \infty}[n]\left(\frac{q_{n}\left(q_{n}+2\right)\left([n]^{2}-[n]\right)}{[3]([n+1]+\beta)^{2}}-1\right) x^{2}+(2 \alpha+2) x-2 x\left(-\frac{1+a+2 \beta}{2} x+\alpha+\frac{1}{2}\right) \\
&= \lim _{n \rightarrow \infty} \frac{[n]}{[3]([n+1]+\beta)^{2}}\left\{q_{n}\left(q_{n}+2\right)[n]^{2}-q_{n}\left(q_{n}+2\right)[n]\right. \\
&\left.-[3]\left(1+q_{n}[n]+\beta\right)^{2}\right\} x^{2}+x+x^{2}(1+a+2 \beta) \\
&= \lim _{n \rightarrow \infty} \frac{[n]}{[3]([n+1]+\beta)^{2}}\left\{\left(q_{n}\left(q_{n}+2\right)-[3] q_{n}^{2}\right)[n]^{2}\right. \\
&\left.-q_{n}\left(q_{n}+2+2[3](1+\beta)\right)[n]-[3](1+\beta)^{2}\right\} x^{2} \\
&+x+x^{2}(1+a+2 \beta)=-\frac{2 a+1}{3} x^{2}+x .
\end{aligned}
$$

The main result of this section is the following Voronovskaja type theorem:

Theorem 5. Let $f^{\prime \prime} \in C[0,1]$ and $q_{n} \in(0,1), q_{n} \rightarrow 1$ and $q_{n}^{n} \rightarrow a, a \in[0,1)$ as $n \rightarrow \infty$. Then we have

$$
\begin{aligned}
\lim _{n \rightarrow \infty}[n]\left(S_{n, q_{n}}^{*(\alpha, \beta)}(f, x)-f(x)\right)= & \left(-\frac{1+a+2 \beta}{2} x+\alpha+\frac{1}{2}\right) f^{\prime}(x) \\
& +\frac{1}{2}\left(-\frac{2 a+1}{3} x^{2}+x\right) f^{\prime \prime}(x) .
\end{aligned}
$$

Proof. From the Taylor's theorem, we have

$$
f(t)=f(x)+(t-x) f^{\prime}(x)+\frac{1}{2}(t-x)^{2} f^{\prime \prime}(x)+\frac{1}{2}(t-x)^{2}\left(f^{\prime \prime}(\xi)-f^{\prime \prime}(x)\right),
$$

where $\xi$ lies between $t$ and $x$.

Applying $S_{n, q_{n}}^{*(\alpha, \beta)}$ on both side of (4.1), we obtain

$[n]\left(S_{n, q_{n}}^{*(\alpha, \beta)}(f, x)-f(x)\right)=[n] f^{\prime}(x) S_{n, q_{n}}^{*(\alpha, \beta)}(t-x, x)$ 


$$
\begin{aligned}
& +\frac{1}{2}[n] f^{\prime \prime}(x) S_{n, q_{n}}^{*(\alpha, \beta)}\left((t-x)^{2}, x\right) \\
& +[n] S_{n, q_{n}}^{*(\alpha, \beta)}\left(\frac{(t-x)^{2}}{2}\left(f^{\prime \prime}(\xi)-f^{\prime \prime}(x)\right), x\right) .
\end{aligned}
$$

For all $x, y \in[0,1],\left|f^{\prime \prime}(\xi)-f^{\prime \prime}(x)\right| \leq \omega_{f^{\prime \prime}}(\delta)\left(1+\frac{(t-x)^{2}}{\delta^{2}}\right)$, for any $\delta>0$.

In view of the Lemma 4 and Lemma 5, we have

$$
\begin{aligned}
& \left|[n] S_{n, q_{n}}^{*(\alpha, \beta)}\left((t-x)^{2}\left(f^{\prime \prime}(\xi)-f^{\prime \prime}(x)\right), x\right)\right| \\
& \leq \omega_{f^{\prime \prime}}(\delta)[n] S_{n, q_{n}}^{*(\alpha, \beta)}\left((t-x)^{2}+\frac{(t-x)^{4}}{\delta^{2}}, x\right)=\omega_{f^{\prime \prime}}(\delta)\left(O(1)+\frac{1}{\delta^{2}} O\left(\frac{1}{[n]}\right)\right) .
\end{aligned}
$$

Choosing $\delta=[n]^{-1 / 2}$, we get

$$
\left|[n] S_{n, q_{n}}^{*(\alpha, \beta)}\left((t-x)^{2}\left(f^{\prime \prime}(\xi)-f^{\prime \prime}(x)\right), x\right)\right|=\omega_{f^{\prime \prime}}\left([n]^{-1 / 2}\right) \cdot O(1) .
$$

Hence

$$
\lim _{n \rightarrow \infty}\left|[n] S_{n, q_{n}}^{*(\alpha, \beta)}\left((t-x)^{2}\left(f^{\prime \prime}(\xi)-f^{\prime \prime}(x)\right), x\right)\right|=0 .
$$

In view of Lemma 5 , we obtain

$$
\begin{aligned}
\lim _{n \rightarrow \infty}[n]\left(S_{n, q_{n}}^{*(\alpha, \beta)}(f, x)-f(x)\right)= & \left(-\frac{1+a+2 \beta}{2} x+\alpha+\frac{1}{2}\right) f^{\prime}(x) \\
& +\frac{1}{2}\left(-\frac{2 a+1}{3} x^{2}+x\right) f^{\prime \prime}(x)
\end{aligned}
$$

\section{NUMERICAL EXAMPLE}

Let us take $f(x)=x^{2}$. In Table 5 we compute error estimation for operators (1.4) and (1.5) to the function $f$.

TABLE 1. Error estimate ( $n=30, \alpha=2, \beta=4, q=0.89)$

\begin{tabular}{|l|l|l|l|l|l|l|l|l|l|l|l|}
\hline$x$ & 0 & 0.1 & 0.2 & 0.3 & 0.4 & 0.5 & 0.6 & 0.7 & 0.8 & 0.9 & 1 \\
\hline$\tilde{S}_{n, q}^{(\alpha, \beta)}$ & 0.0393 & 0.0631 & 0.0743 & 0.0730 & 0.0590 & 0.0325 & 0.0067 & 0.0584 & 0.1227 & 0.1996 & 0.2890 \\
\hline$S_{n, q}^{*(\alpha, \beta)}$ & 0.0393 & 0.0638 & 0.0763 & 0.0768 & 0.0652 & 0.0417 & 0.0062 & 0.0412 & 0.1007 & 0.1722 & 0.2556 \\
\hline
\end{tabular}

For $n=30, \alpha=2, \beta=4, q=0.89$, the convergence of operators (1.4) and (1.5) to function $f(x)=x^{2}$ is illustrated in Figure 1 . 


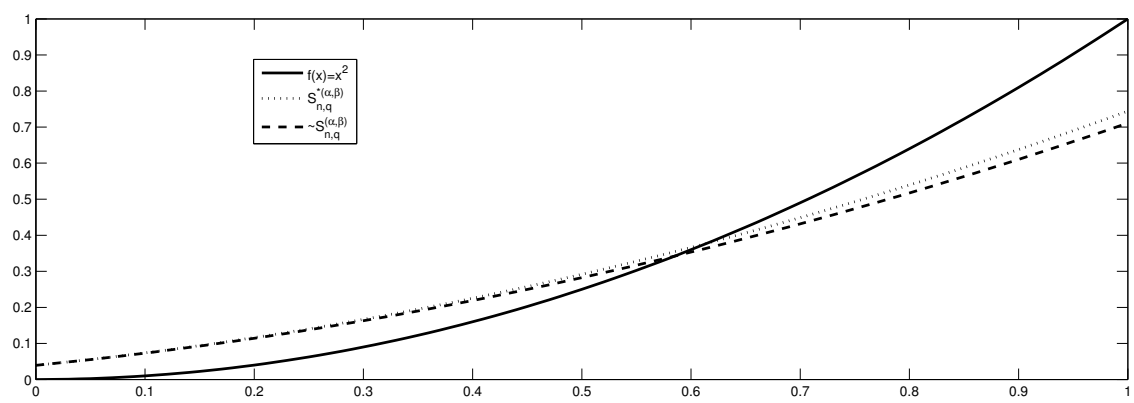

FIGURE 1. Function $f(x)=x^{2}$ and $q$-Stancu-Kantorovich operators.

\section{REFERENCES}

[1] V. Cleciu, "Approximation properties of a class of Bernstein-Stancu type operators," in Proceedings of the international conference NAAT, O. Agratini and P. Blaga, Eds. Cluj-Napoca: Casa Cartii de Stiinta, 2006, pp. 171-178.

[2] V. Cleciu, "Bernstein-Stancu operators," Studia Univ.Babes-Bolyai, Mathematica, vol. 52, no. 4, pp. 53-65, 2007.

[3] O. Dalmanoğlu, "Approximation by Kantorovich type $q$-Bernstein operators," in Proceedings of the 12th WSEAS Int. Conf. on Applied Mathematics, M. Demiralp, C. Udriste, G. Bognar, R. Soni, and H. Nassar, Eds. Cairo, Egypt: WSEAS Press, 2007, pp. 29-31.

[4] O. Dalmanoğlu and O. Doğru, "On statistical approximation poperties of Kantorovich type $q$ Bernstein operators," Mathematical and Computer Modelling, vol. 52, pp. 760-771, 2010.

[5] O. Doğru and V. Gupta, "Korovkin-type approximation properties of bivariate q-Meyer-König and Zeller operators," Calcolo, vol. 43, no. 1, pp. 51-63, 2012.

[6] V. Gupta and H. Wang, "The rate of convergence of q-Durrmeyer operators for $0<q<1$," Math. Methods Appl. Sci., vol. 31, no. 16, pp. 1946-1955, 2008.

[7] A. Lupaş, "A q-analogue of the Bernstein operator," in Seminar on Numerical and Statistical Calculus, G. Coman and I. Rus, Eds. Cluj-Napoca: ”Babes-Bolyai” University, 1987, pp. 85-92.

[8] N. Mahmudov and P. Sabancigil, "Approximation theorems for $q$-Bernstein-Kantorovich operators," Filomat, vol. 27, no. 4, pp. 721-730, 2013.

[9] S. Marinković, P. Rajković, and M. Stanković, "The inequalities for some types of q-integrals," Comput. Math. Appl., vol. 56, no. 10, pp. 2490-2498, 2008.

[10] C. Muraru, "Note on q-Bernstein-Schurer operators," Studia Univ.Babes-Bolyai, Mathematica, vol. 56, no. 2, pp. 1-11, 2011.

[11] C. Muraru, "Note on the q-Stancu-Schurer operator," Miskolc Mathematical Notes, vol. 14, no. 1, pp. 191-200, 2013.

[12] M. A. Özarslan and T. Vedi, "q-Bernstein-Schurer-Kantorovich operators," Journal of Inequalities and Applications, vol. 2013:444, no. doi:10.1186/1029-242X-2013-444, 2013.

[13] G. Philips, "On generalized Bernstein polynomials," in Numerical Analysis, D. F. Griffits and G. A. Watson, Eds. Singapore: World Scientific, 1996, pp. 263-269.

[14] M. Ren and X. M. Zeng, "On statistical approximation properties of modified q-Bernstein-Schurer operators," Bull. Korean Math. Soc., vol. 50, no. 4, pp. 1145-1156, 2013. 
[15] M. Ren and X. M. Zeng, "Some statistical approximation properties of Kantorovich-type $q$ Bernstein-Stancu," Journal of Inequalities and Applications, vol. 2014:10, no. doi:10.1186/1029242X-2014-10, 2014.

[16] D. Verma and P. Agrawal, "Approximation by Baskakov-Durrmeyer-Stancu operators based on q-integers," Lobachevskii J. Math., vol. 43, no. 2, pp. 187-196, 2013.

Authors' addresses

Ana Maria Acu

Lucian Blaga University of Sibiu, Department of Mathematics and Informatics, Str. Dr. I. Ratiu, No.5-7, RO-550012 Sibiu, Romania

E-mail address: acuana77@yahoo.com

\section{Dan Bărbosu}

Technical University of Cluj-Napoca, North University Center at Baia Mare, Department of Mathematics and Computer Science, Str. Victoriei 76, RO-430122 Baia Mare, Romania

E-mail address: danbarbosulyahoo.com

\section{Daniel Florin Sofonea}

Lucian Blaga University of Sibiu, Department of Mathematics and Informatics, Str. Dr. I. Ratiu, No.5-7, RO-550012 Sibiu, Romania

E-mail address: sofoneaflorin@yahoo.com 